\title{
BOUNDED RENORMALIZATION WITH CONTINUOUS PENALIZATION FOR LEVEL SET INTERFACE CAPTURING METHODS
}

\author{
Laura Battaglia ${ }^{\mathrm{a}, \mathrm{b}}$, Mario A. Storti ${ }^{\mathrm{a}}$ and Jorge D'Elía ${ }^{\mathrm{a}}$ \\ ${ }^{a}$ Centro Internacional de Métodos Computacionales en Ingeniería (CIMEC), Instituto de Desarrollo \\ Tecnológico para la Industria Química (INTEC), Universidad Nacional del Litoral - CONICET, \\ Güemes 3450, 3000-Santa Fe, Argentina, e-mail: lbattaglia@santafe-conicet.gob.ar,web page: \\ http://www.cimec.org.ar \\ ${ }^{\mathrm{b}}$ Grupo de Investigación en Métodos Numéricos en Ingeniería (GIMNI), Universidad Tecnológica \\ Nacional, Facultad Regional Santa Fe, Lavaisse 610, 3000-Santa Fe, Argentina
}

Keywords: interface-capturing, level-set method, finite element method, bounded renormalization, continuous penalization

\begin{abstract}
In this work, a reinitialization procedure oriented to regularize the Level Set (LS) function field is presented. In LS approximations for two-fluid flow simulations, a scalar function indicates the presence of one or another phase and the interface between them. In general, the advection of such function produces a degradation of some properties of the LS function, such as the smoothness of the transition between phases and the correct position of the interface. The methodology introduced here, denominated bounded renormalization with continuous penalization, consists of solving by the finite element method a partial differential equation with certain distinguishing properties with the aim of keeping the desirable properties of the LS function. The performance of the strategy is evaluated for several typical cases in one, two and three-dimensional domains, for both the advection and the renormalization stages.
\end{abstract}




\section{INTRODUCTION}

It is well known that there are two main approaches for the computation of two-phase or free surface flows: interface tracking or interface capturing Shyy et al. (1996). The first approach consists of following explicitly the interface because it is defined over discrete entities such as nodes or elements of a finite element mesh. An interface capturing strategy generally counts on a fixed or Eulerian mesh where the frontier between fluids crosses the elements and must be captured by an additional component, such as an auxiliary variable or a fluid fraction.

On one side, interface tracking methods can be purely Lagrangian, as particle methods Idelsohn et al. (2004), or they are developed as Arbitrary Lagrangian-Eulerian (ALE) approaches Hughes et al. (1981); Huerta and Liu (1988); Battaglia et al. (2006), where the interface is defined over grid entities such as facets or edges. Then, the interface movement produces the displacement of such entities and, in ALE approximations, the mesh must be deformed or regenerated in order to take into account that deformation. By the other side, interface capturing methods as volume of fluid (VOF) Hirt and Nichols (1981); Scardovelli and Zaleski (1999) or level set (LS) Osher and Sethian (1988); Sethian (1995) were developed for modelling topological changes like merging or breaking up of the interfaces involved, and both of them require an additional parameter for detecting the presence of the interface in an Eulerian referential. Particularly, LS methods are not limited to incompressible flows. It is also used for simulating crystal growth Osher and Fedkiw (2001), in image processing Osher and Fedkiw (2001); Gloger et al. (2009), the material distribution within microstructure in solid mechanics Hettich and Ramm (2006), the interaction of a compressible high-speed flow and a highly flexible thin-shell structure Cirak and Radovitzky (2005), or computational biophysics Maitre et al. (2009), among other phenomena. Regarding advantages and disadvantages of classic numerical methods for interface simulations, hybrid methodologies arised with the aim of improving the results. Some examples are the combined LS and VOF method (CLSVOF) from Sussman and Puckett Sussman and Puckett (2000), the Particle Level Set from Enright et al. Enright et al. (2002), or the combination of front tracking and front capturing techniques, as the one presented by Shin and Juric Shin and Juric (2009).

In LS interface capturing approaches, for the general case of two-fluid flows, each phase is usually indicated with a positive or negative value of the LS function $\phi$, being $\phi>0$ for one phase, $\phi<0$ for the other one, and $\phi=0$ is the level set corresponding to the interface position Osher and Sethian (1988). The LS function $\phi$ is defined over the whole domain of analysis, is continuous across the interface $\phi=0$ and is used for indicating the fluid properties when the flow problem is numerically solved. According to the physics of the problem, the LS function evolves in time following an advection equation, where the advective velocity depends on the problem to be solved Osher and Fedkiw (2001). This kind of approximation counts on numerous variants which were generated in order to avoid some problems associated to the advection of the LS function $\phi$ Sussman and Smereka (1997), such as volume variation in one of the phases involved and the loss of the smoothness of the transition between fluids. Then, some approaches work on the advection scheme for $\phi$ to avoid these problems, by applying high order integration schemes as essentially non oscillatory (ENO) and weighted-ENO (WENO) Osher and Sethian (1988); Osher and Fedkiw (2001), or discontinuous Galerkin methods Marchandise 
and Remacle (2006); Di Pietro et al. (2006).

Other LS approaches are complemented by a reinitialization for the $\phi$ field in order to keep their smooth properties and, in some cases, compensate or avoid the volume loss at the same time Sussman and Smereka (1997); Mut et al. (2006). Generally speaking, reinitialization of the LS function $\phi$ field consists of a redistancing procedure where the $\phi$ values are determined as the distance of each point to the $\phi=0$ curve. This can be done through brute force algorithms, which are extremely expensive Elias et al. (2007), by solving similar problems related to the distance to the interface Sussman and Smereka (1997); Elias et al. (2007); Mut et al. (2006), or by proposing an auxiliary advection-diffusion like problem Olsson and Kreiss (2005); Olsson et al. (2007), among others.

In this work, an advection-renormalization strategy for an LS method is presented. The algorithm consists of two stages solved alternately: the first one is a typical advection stage, which determines the transport of the $\phi$ function due to the application of a velocity field; the second one, which can be done every certain number of temporal steps, keeps the regularity of the LS function $\phi$, mainly in the neighbourhood of the interface. The renormalization stage, denominated as bounded renormalization with continuous penalization (BRCP), consists of solving a partial differential equation with particular properties, leading to a smooth, continuous, bounded LS field such that it allows the resolution of problems like two-fluid flows, where the properties of each fluid are interpolated across a narrow strip near the interface throughout certain functions of the LS field. Part of this strategy was introduced before for solving free surface fluid flows Battaglia (2009); Battaglia et al. (3-6 November 2009), though only the aspects that concern the transport of the LS function $\phi$ and its reinitialization by BRCP are considered here. Each instance is numerically solved by a finite element method (FEM) through the use of libraries from the PETSc-FEM code PETSc-FEM (2009), which is based on the Portable Extensible Toolkit for Scientific Computation (PETSc) Balay et al. (2008), and the Message Passing Interface (MPI) libraries for parallel computing MPI (2009).

The LS formulation is described in Section 2, including the LS field advection problem, and a brief description of a renormalization method that precedes the one introduced in this work is described in Section 3. Then, the advection-renormalization methodology is evaluated in Section 4 for typical problems found in literature for two- and three-dimensional cases, whereas one-dimensional examples are introduced for discussing the renormalization parameters to be taken into account. Finally, Section 5 includes the concluding remarks.

\section{LS FORMULATION}

\subsection{LS function}

The LS function $\phi=\phi(\mathbf{x}, t)$ represents a continuous field defined over the whole domain of analysis $\Omega$, and is given for the position $\mathrm{x} \in \Omega$ at time $t$ in the case of transient problems. The evolution of the $\phi$ field is governed by the physical phenomena involved in the simulation, e.g. by the fluid velocity $\mathbf{v}(\mathbf{x}, t)$ in the case of two-fluid flows Osher and Fedkiw (2001).

The LS scalar field describes which part of $\Omega$ is occupied by one phase or the other, and 
particularly where the interface is placed between them, in the following way,

$$
\phi(\mathbf{x}, t)\left\{\begin{array}{lll}
>0 & \text { if } & \mathbf{x} \in \Omega_{1} \\
=0 & \text { if } & \mathbf{x} \in \Gamma_{\mathrm{I}} \\
<0 & \text { if } & \mathbf{x} \in \Omega_{2}
\end{array}\right.
$$

where $\Omega_{1}$ and $\Omega_{2}$ are the subdomains occupied by one or the other phase, which verify $\Omega=$ $\Omega_{1} \cup \Omega_{2}$ and $\Omega_{1} \cap \Omega_{2}=\varnothing$. The interface is captured as

$$
\Gamma_{\mathrm{I}}=\{\mathbf{x} \mid \phi(\mathbf{x}, t)=0\}
$$

Regarding the method proposed for the renormalization of $\phi$ in Section 3, the LS function verifies $-1 \leq \phi \leq 1$, with a smooth continuous transition between these values.

\subsection{Advection of the LS field}

The transport of the LS function $\phi$ over the domain $\Omega$ is generated by the velocity field $\mathbf{v}$ given by the phenomena simulated, as the Navier-Stokes equations in the case of viscous and incompressible two fluid flows, and can be written as follows,

$$
\partial_{t} \phi+\mathbf{v} \cdot \nabla \phi=0
$$

where $\partial_{t}(\ldots)=\partial(\ldots) / \partial t$ indicates the partial time derivative and the boundary conditions over the frontiers of the domain $\Gamma$ are given by

$$
\phi=\bar{\phi} \quad \text { over } \quad \Gamma_{\text {in }}
$$

where inflow sections are $\Gamma_{\text {in }}=\{\Gamma \mid \mathbf{v} \cdot \mathbf{n}<0\}$, with $\mathbf{n}$ being the outgoing unit normal of the boundary. This advection procedure takes into account the transport of the interface $\Gamma_{\mathrm{I}}$ in a natural way. Moreover, in the one-dimensional cases presented in Section 4.1, periodic boundary conditions are used.

The numerical resolution of this advection problem is made by FEM with some of the libraries of the PETSc-FEM code PETSc-FEM (2009). Since the Galerkin formulation is used for the resolution of the transport of $\phi$ given by Equation (3), the typical numerical instabilities should be avoided, e.g. by using the streamline upwind/Petrov-Galerkin (SUPG) strategy Brooks and Hughes (1982); Tezduyar and Osawa (2000).

\subsection{Simultaneous advection-renormalization}

Instead of the pure advection problem given in Equation (3), other formulation was proposed in Battaglia et al. (10-13 November 2008) and Battaglia (2009), with the aim of renormalizing the LS function $\phi$ at the same time the $\phi$ field is advected, avoiding the use of a renormalization stage. Then, two additional terms were introduced at the right hand side of the $\phi$ advection equation,

$$
\partial_{t} \phi+\mathbf{v} \cdot \nabla \phi=\underbrace{C \phi\left(\phi^{2}-\phi_{\mathrm{ref}}^{2}\right)}_{\mathcal{R}}-\underbrace{\kappa(\phi) \Delta \phi}_{\mathcal{D}}
$$


while the boundary conditions are the same as in Equation (4). The arbitrary positive constant $C$, or regularizing parameter, is given in time ${ }^{-1}$ units. Numerical tests with $0<C \leq 10 \mathrm{~s}^{-1}$ have not shown neither numerical instabilities nor severe precision loss. Results showed that $C=10 \mathrm{~s}^{-1}$ provided the higher error in $\Gamma_{\mathrm{I}}$ position, while $C=1 \mathrm{~s}^{-1}$ gave an acceptable performance Battaglia et al. (10-13 November 2008). Note that in case $C=0$, Equation (3) is recovered.

The Laplacian operator is $\Delta$, and $\kappa(\phi)$ is a diffusive parameter determined as follows,

$$
\kappa(\phi)=\kappa_{\text {ref }} \min \left(|\phi| / \phi_{\text {ref }}, 1\right)
$$

with the reference value for the variable $\phi_{\text {ref }}=1$ and $\kappa_{\text {ref }}$ proposed such as

$$
\kappa_{\text {ref }}=\varepsilon^{2} C
$$

in length ${ }^{2} /$ time units. The length $\varepsilon$ is given by the user, and represents the width of half the transition strip between the bounds $\pm \phi_{\text {ref }}$, in such a way that $\varepsilon / h$ establishes the quantity of elements in one half of this transition strip, being $h$ a typical element size.

This reaction-diffusion formulation on the right hand side of Equation (5) was proposed for reproducing a behaviour similar to those found in combustion phenomena Logan (1994), where the roots $\phi= \pm \phi_{\text {ref }}$ of $\mathcal{R}$ give the stable "on/off" states, while the diffusive term $\mathcal{D}$ provides a continuous smooth transition between them. The remaining root $\phi=0$ in $\mathcal{R}$ ensures that this term is null for the transport of the zero LS, as well as in $\mathcal{D}$, where from Equation (6) results $\kappa(0)=0$. Then, when $\phi$ tends to zero, Equation (3) is recovered, and the position of the interface $\Gamma_{\mathrm{I}}$ should not be affected by these additional terms, at least in a continuous formulation.

The problem given by Equation (5) is numerically solved by FEM, considering the SUPG strategy for the advective terms in case numerical stabilization was needed, as mentioned in Section 2.2.

The results obtained through this strategy showed that the transition strip width was not constant, and some results were more diffusive and less precise than expected Battaglia et al. (10-13 November 2008); Battaglia (2009). As a consequence, the simultaneous advectionrenormalization stage was divided into separated procedures, keeping the idea of a continuous operator as the base for the reinitialization procedure of Section 3.

\section{REINITIALIZATION OF THE LS FUNCTION}

The reinitialization or renormalization procedure presented here was introduced in Battaglia (2009), and consists of the resolution of a PDE system by FEM, where the variable is the LS function $\phi$. This procedure is a consequence of the methodology accounted in Section 2.3, where advection and renormalization of the LS function were solved at the same time. A similar approach is given in Olsson and Kreiss Olsson and Kreiss (2005), and Olsson et al. Olsson et al. (2007) as part of the so-called Conservative Level Set Method, although the reinitialization procedure given in those references lacks of penalization term.

The operator to be applied after solving Equation (3), denominated BRCP and presented in this work, is proposed as

$$
\phi\left(\phi^{2}-\phi_{\text {ref }}^{2}\right)-\kappa \Delta \phi+M\left[\check{H}(\phi)-\check{H}\left(\phi^{0}\right)\right]=0
$$


being $\kappa$ a diffusive parameter or diffusivity, $M$ a penalty coefficient, and $\phi_{\text {ref }}$ a reference value for the variable $\phi$, in this case adopted as $\phi_{\text {ref }}=1$, whereas $\phi^{0}$ is the initial LS function value for the renormalization step provided by the solution of Equation (3). The diffusive parameter $\kappa$ is given in length ${ }^{2}$ units, and is related to a typical element size $h$ for the mesh, usually from $h^{2}$ to $(3 h)^{2}$, depending on the interface behaviour: a lower $\kappa$ provides a thinner transition.

The penalizing term of Equation (8), where the penalty coefficient $M$ is used, allows taking into account the known values $\phi^{0}$, i.e. those determined in the advection stage. The aim of this term is avoiding the displacement of the interface, represented by the zero LS $\phi=0$, by weighting $\check{H}(\phi)-\check{H}\left(\phi^{0}\right)$. In this term, the penalty coefficient $M$ is positive and nondimensional, and the penalization function $\breve{H}(\phi)$ is chosen as the continuous $\left(C^{\infty}\right)$ expression

$$
\check{H}(\phi)=\tanh (2 \pi \phi) .
$$

The choice of such function for the penalization term is motivated by the advantages of using a smooth, bounded, infinitely differentiable function against discontinuous ones, as the classical Heaviside function, which would require special numerical treatment, particularly in three-dimensional problems. The argument of the function in Equation (9) is chosen for concentrating the effect of the penalizing term nearby the interface, e.g. the penalty term at half the transition from $\phi=0$ and $\phi=1$ is $\check{H}(0.5)-\check{H}(0.49)=5 \times 10^{-4}$, whereas it is $\check{H}(0.1)-\check{H}(0.09)=4.5 \times 10^{-2}$ close to the interface. In coupled advection-renomalization problems, convenient values for the penalization $M$ are $\mathcal{O}\left(10^{n_{\mathrm{d}}+2}\right)$ with $n_{\mathrm{d}}$ the number of spatial dimensions of the problem considered, as will be shown in Section 4. Low values for $M$, such as $M=1$, lead to high error in the $\Gamma_{\mathrm{I}}$ position, and $M=0$ makes the algorithm fail because it lacks the reference $\phi^{0}$ from the advective step. For values of $M$ higher than $\mathcal{O}\left(10^{n_{\mathrm{d}}+2}\right)$, the renormalization effect is lost, because the $\phi$ field tends to the $\phi^{0}$ field, i.e., the solution obtained with pure advection is recovered. Other interface capturing method with penalty parameter for preserving the interface position is the edge-tracked interface locator technique (ETILT) method Tezduyar (2006); Cruchaga et al. (2007).

As an example of how the first two terms of Equation (8) act over the $\phi$ field, their effect will be analysed over a one-dimensional domain $0 \leq x \leq L$,

$$
C_{r} \phi\left(\phi^{2}-\phi_{\text {ref }}^{2}\right)-\kappa \Delta \phi=0
$$

which is a stationary reaction-diffusion-like equation, where $\phi_{\text {ref }}$ is a given value and $C_{r}$ is a non-dimensional regularizing coefficient, different from the one in Equation (5) because this problem is stationary. Dirichlet boundary conditions are given.

For analysing the behaviour of each term in Equation (10), the Laplacian part is eliminated with $\kappa=0$; then, the roots of the remaining reactive equation are $\phi= \pm \phi_{\text {ref }}= \pm 1$ and $\phi=0$, and there are infinite solutions, which are piecewise-constant, and where each segment takes one of the roots values. If the diffusivity $\kappa$ is reintroduced, each pair of segments of the contiguous solution for $\kappa=0$ is smoothly connected in a continuous way, as seen in Figure 1.

The behaviour of the roots of the reactive term of Equation (10) is analysed throughout an analogy with a steady heat conduction equation with a nonlinear source term, where $\phi$ repre- 
sents the temperature. Effectively, this equation can be put in the form

$$
0=\kappa \Delta \phi+Q(\phi)
$$

where there are a source term $Q(\phi)=-C_{r} \phi\left(\phi^{2}-\phi_{\text {ref }}^{2}\right)$ and a conduction term with a thermal diffusivity $\kappa$. Physically, the source term $Q(\phi)$ tends to force $\phi$ to reach the stable equilibrium temperatures, i.e. those $\phi^{*}$ for which

$$
Q\left(\phi^{*}\right)=0, \text { and } Q^{\prime}\left(\phi^{*}\right)<0
$$

where $Q^{\prime}(\phi)=-C_{r}\left(3 \phi^{2}-\phi_{\text {ref }}^{2}\right)$. Owing to the first condition of Equation (12), the source term is null at these temperatures, so that a spatially constant solution $\phi=\phi^{*}$ is a solution of Equation (11). Now, if $\phi$ changes slightly from $\phi^{*}$, e.g. $\phi=\phi^{*}+\delta \phi$ with $\delta \phi>0$, then the source term becomes negative due to the second condition in Equation (12), so that it tends to bring $\phi$ back to $\phi^{*}$, and the same occurs if $\delta \phi<0$. This condition is analogous for the roots $\phi^{*}= \pm \phi_{\text {ref }}$ of Equation (10). Conversely, for those roots that satisfy

$$
Q\left(\phi^{*}\right)=0, \text { and } Q^{\prime}\left(\phi^{*}\right)>0
$$

the feedback given by the source term is positive, so that it tends to take away $\phi$ from the equilibrium value $\phi^{*}$, and it is an unstable equilibrium temperature, which is analogous for $\phi^{*}=0$ in Equation (10).

In LS methods, the key is the definition of the regions occupied by one or another phase, which correspond to non-null values for the LS function $\phi$, and the interface is an entity of one dimension less than the spatial domain studied; other cases are meaningless in this context. Then, regarding Equation (10), if the initial conditions for the reinitialization process takes $\phi$ values different from zero, or at most takes this value in one point between $\phi>0$ and $\phi<0$, then the instability of the near-zero region will provide that the zero LS will be crossed by the solution curve at most on one point for one dimensional problem, over a line for two dimensional ones, or over a surface in three dimensional cases.

See for example Figure 1 for the case $\kappa=0$, where solutions of Equation (10) are constant and take values $\phi= \pm \phi_{\text {ref }}$. If the diffusivity $\kappa$ is reintroduced, each pair of segments of the contiguous solution for $\kappa=0$ is smoothly connected in a continuous way, as seen also in the same figure.

The transition width from $-\phi_{\text {ref }}$ to $\phi_{\text {ref }}$ is given by

$$
\gamma=\mathcal{O}\left(\sqrt{\frac{\kappa}{C_{r}}}\right)
$$

Then, by adopting $C_{r}=1$ for all the cases, the LS function transition strip is governed by the diffusivity $\kappa$. For appreciating this, in Figure 1 there are two solutions for two different values of $\kappa$, where the higher one provides a wider transition.

\section{0 truecm}

Regarding the choice of the free parameter values, diffusivity $\kappa$ and penalization $M$, in cases where the interface would suffer breaking up or would fold several times, it is convenient to 


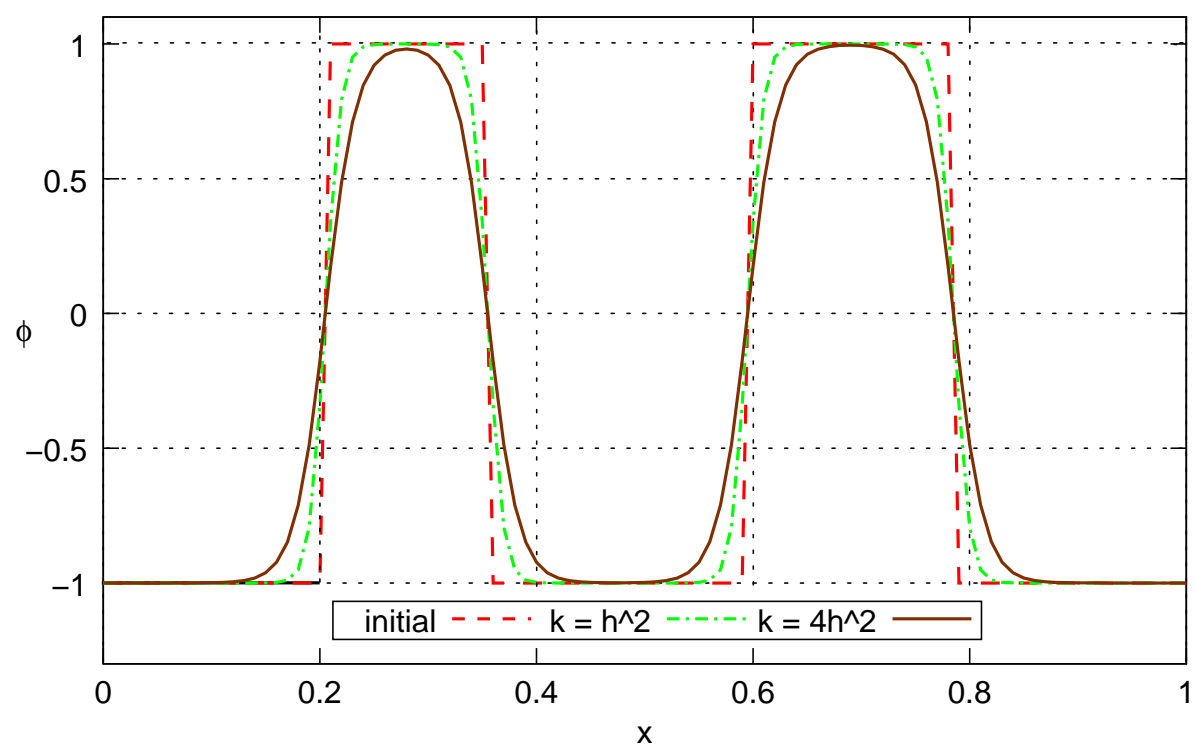

Figure 1: Artificial reaction-diffusion problem for renormalization of $\phi$ depending on the diffusion coefficient $\kappa$ values.

adopt a low $\kappa$ value and a high $M$ one, because transitions should be thinner and $M$ would avoid the disappearance of the drops.

The regions placed far from the interface are not affected by the renormalization process because, when $\phi \rightarrow \phi_{\text {ref }}$, then all the three terms in Equation (8) tends to zero. On the other hand, the higher influence of the operator from Equation (8) is registered in the neighbourhood of $\phi=0$, where the lower precision in the interface position and mass loss is observed Mut et al. (2006); Cruchaga et al. (2007).

The operator presented in Equation (8) is numerically solved as a steady problem by a standard FEM, taking as initial condition $\phi^{0}$ the $\phi$ values transported by the previous advection step. Besides, the regularization can be applied each time step or every $n_{\text {reno }}$ time steps.

\section{NUMERICAL EXAMPLES}

A battery of numerical tests is proposed in order to show the advantages of the application of the BRCP strategy for renormalization of LS-like functions $\phi$. These examples will be solved in two ways:

- advection, with or without SUPG stabilization;

- advection plus renormalization;

which are named ADV and ADV+BRCP, respectively, in order to shorten the description of the examples. The performances of both strategies are compared and the application field for BRCP is established. Linear finite elements are used in all the cases, for both stages of resolution.

\subsection{One-dimensional fields}

For illustrating the effect of the advection-renormalization sequence and the selection of appropriate values for the diffusivity $\kappa$ and the penalty parameter $M$ in BRCP, two one-dimensional 


\begin{tabular}{|l|c|c|c|c|} 
Elapsed time & \multicolumn{2}{|c|}{$t=1 \mathrm{~s}$} & \multicolumn{2}{c|}{$t=3 \mathrm{~s}$} \\
Numerical method and mesh & $\Delta_{0}[\mathrm{~m}]$ & $\Delta l[\mathrm{~m}]$ & $\Delta_{0}[\mathrm{~m}]$ & $\Delta l[\mathrm{~m}]$ \\
ADV & $8.4 \times 10^{-3}$ & $1.5 \times 10^{-2}$ & $4.3 \times 10^{-2}$ & $8.0 \times 10^{-2}$ \\
ADV+BRCP, $\kappa=2 h^{2}$ & $4.9 \times 10^{-3}$ & $6.8 \times 10^{-3}$ & $1.0 \times 10^{-2}$ & $7.1 \times 10^{-3}$ \\
ADV+BRCP, $\kappa=4 h^{2}$ & $5.6 \times 10^{-3}$ & $9.2 \times 10^{-3}$ & $1.5 \times 10^{-2}$ & $2.1 \times 10^{-2}$
\end{tabular}

Table 1: Maximum zeros displacements $\Delta_{0}$ and variation of the distance between consecutive zeros of $\phi, \Delta l$, for the sinusoid problem after one lap $(t=1 \mathrm{~s})$ and three laps $(t=3 \mathrm{~s})$ around the periodic domain.

problems are considered. Both are solved over a domain of length $L=1 \mathrm{~m}$, divided in 100 finite elements of length $h=0.01 \mathrm{~m}$ and considering an advection velocity of $v=1 \mathrm{~m} / \mathrm{s}$.

Other common issues for the following examples are the frequency of renormalization, every $n_{\text {reno }}=10$ time steps, and the use of periodic boundary conditions between $x=0$ and $x=L$. Then, for each lap around the periodic domain, for example for a final time $t_{f}=1 \mathrm{~s}$, the advected field should be equal to the initial one, at least regarding the $\phi=0$ coordinate, which indicates the position of the interface in higher dimensional problems.

The time step adopted is $\Delta t=0.005 \mathrm{~s}$, giving in all the cases a Courant number Co $=0.5$, which implies that the advective step will provide stable and a little diffusive solutions. Besides, the temporal integration in the solution of the advection problem is implicit due to the adoption of an integration coefficient $0.7<\theta \leq 1.0$ for the trapezoidal rule Donea and Huerta (2003). It should be noted that solutions with numerical stabilization are explicitly indicated.

\subsubsection{Sinusoid}

The first example consists of the evaluation of a travelling sinusoid solved by two alternatives, $\mathrm{ADV}$ and $\mathrm{ADV}+\mathrm{BRCP}$, represented together with the initial condition in Figure 2, given by

$$
\phi(x)=\sin (s+6 \pi x)+0.30
$$

for $0 \leq x \leq(L-0.01 \mathrm{~m})$. The shift $s=0.01 \mathrm{~m}$ in $x$ direction is used for avoiding problems at the measurement of the position of $\phi=0$ in case the wave suffers a delay in the displacements for the periodic domain. Moreover, the displacement in $\phi$ of 0.30 is introduced in order to reach an initial condition with different sizes of positive and negative region lengths in the $x$-axis, regarding the LS function value, i.e., $\phi>0$ or $\phi<0$, respectively.

\section{2 truecm}

The resolution is performed with an integration coefficient $\theta=0.7$ for the time integration with the trapezoidal rule, and the purpose of this example is showing the influence of the diffusive parameter $\kappa$ over the resulting curves, as well as the volume preservation properties of the BRCP scheme.

12 truecm

The solution obtained with ADV shows a loss of amplitude, especially in the negative peaks, and the zeros positions are displaced, see Figure 2. Such displacement, which is not present in $\mathrm{ADV}+\mathrm{BRCP}$ cases, is due to the symmetry of the curve with respect to the mean value $\phi=0.30$ computed with ADV. On the contrary, BRCP solutions are obtained in such a way that the length 


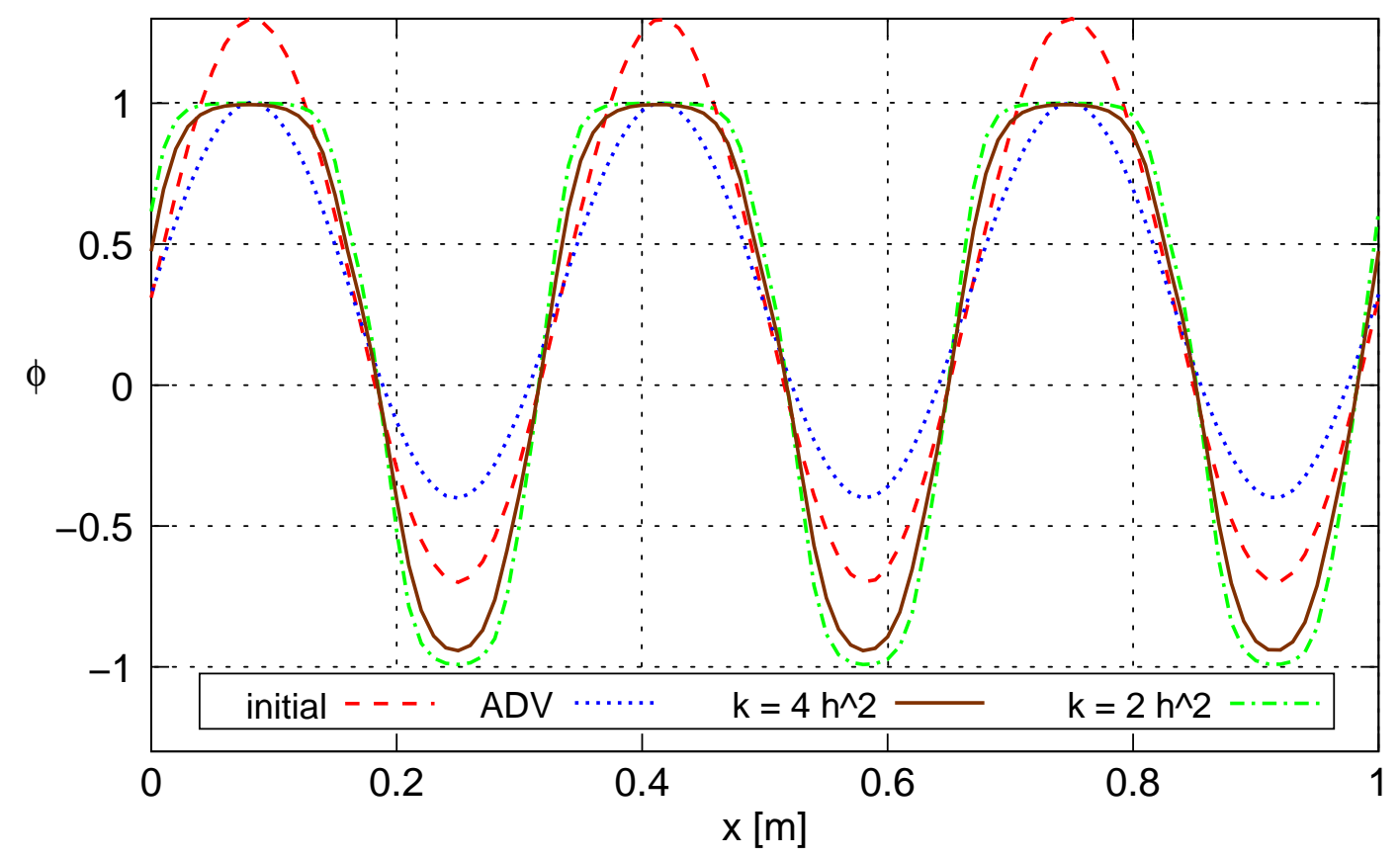

Figure 2: Travelling sinusoid solved by ADV, ADV+BRCP for different values of diffusivity $\kappa$, and the initial condition.

of the positive and negative regions in $x$-direction is kept independently of the amplitude in $\phi$ direction. Then, when fields with different sizes and shapes of positive and negative regions are solved, the strategy ADV+BRCP performs better than the ADV without reinitialization. Moreover, the results obtained with ADV+BRCP tends to maintain the amplitude forced by the renormalization operator, i.e. $\phi= \pm 1$, particularly for $\kappa=2 h^{2}$, although the peaks are a little lower in the case $\kappa=4 h^{2}$. This difference between the two of the BRCP solutions is due to a higher diffusivity $\kappa$ induces a wider transition between $-\phi_{\text {ref }}$ and $+\phi_{\text {ref }}$, and in this example the transitions are superimposed because of the narrowness of the initial condition in negative peaks. Both of the BRCP final states were determined with penalization $M=1000$.

\section{3 truecm}

The maximum zeros displacements $\Delta_{0}$ for the solutions plotted in Figure 2 are included in Table 1, as well as the variation of the length of the segments defined between zeros $\Delta l$, regarding either positive or negative value for the LS function $\phi$, after one lap ( $t=1 \mathrm{~s}$ ) and three laps $(t=3 \mathrm{~s})$ around the periodic domain, for a uniform mesh. Through this table, it is possible to confirm that the zeros tracking error is higher for ADV than for the ADV+BRCP alternatives, and that the renormalized cases show a better length preservation, regarding the $\Delta l$ column values, with one order of magnitude of difference, particularly for BRCP with $\kappa=2 h^{2}$. The evolution of the maximum displacements of the $\phi=0$ positions, $\Delta_{0}$, is represented in Figure 3 as a function of time, for the solutions obtained with ADV, and with ADV+BRCP considering $\kappa=2 h^{2}$, where the tendency of such error is always higher for ADV. This is due to the loss of amplitude of the ADV solution, which also tends to the disappearance of the regions of $\phi<0$ as the problem evolves in time, whereas BRCP avoids it.

11 truecm

This example is also solved over a non-uniform node distribution randomly generated, with 


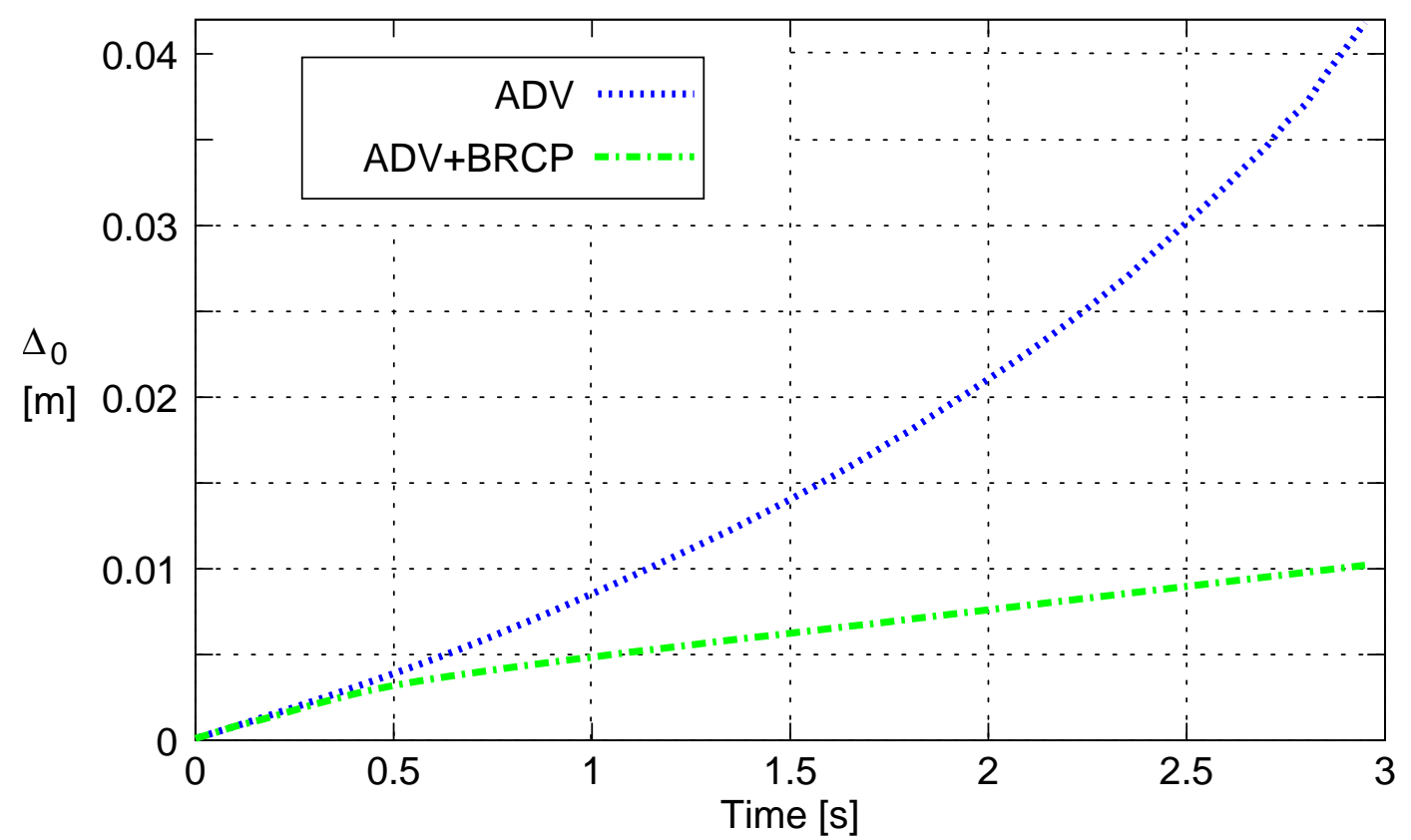

Figure 3: Variation in time of the maximum zeros displacements $\Delta_{0}$ for the resolution with ADV, and ADV+BRCP in the case of the travelling sine function.

a mean $h$ value which corresponds to the element size from the previous analysis, showing a maximum element size of $h_{\mathrm{M}}=0.0208 \mathrm{~m}$ and a minimum one of $h_{\mathrm{m}}=0.0004 \mathrm{~m}$. The curves obtained with ADV+BRCP for the same reinitialization parameters with uniform mesh, i.e. diffusivity $\kappa=2 h^{2}$ and penalization $M=1000$, are practically superimposed for both the uniform and non-uniform meshes, showing the proportionality between the diffusivity $\kappa$ and the transition width, see Figure 4.

\subsubsection{A normalized cardinal sine like function}

The effects of the penalization term from Equation (8) is shown through a symmetric example with three steps, considering a time integration coefficient $\theta=0.7$ and two different mesh sizes, $h=0.01 \mathrm{~m}$ and $h^{\prime}=h / 2=0.005 \mathrm{~m}$. The LS function field is generated in two stages. First, a cardinal sine like function is proposed,

$$
\tilde{\phi}=\frac{\sin [7 \pi(x-f)]}{2 \pi(x-f)}
$$

with $f=0.50 \mathrm{~m}$ the displacement of the function vertex in $x$-direction. The field given by Equation (16) is later normalized in order to work with $\phi$ values inside the bounds proposed by the BRCP method, as follows,

$$
\phi=\operatorname{sign}(\tilde{\phi})
$$

where the sign function is defined as:

$$
\operatorname{sign}(\phi)= \begin{cases}-1 & \text { for } \phi<0 \\ 0 & \text { for } \phi=0 \\ 1 & \text { for } \phi>0 .\end{cases}
$$




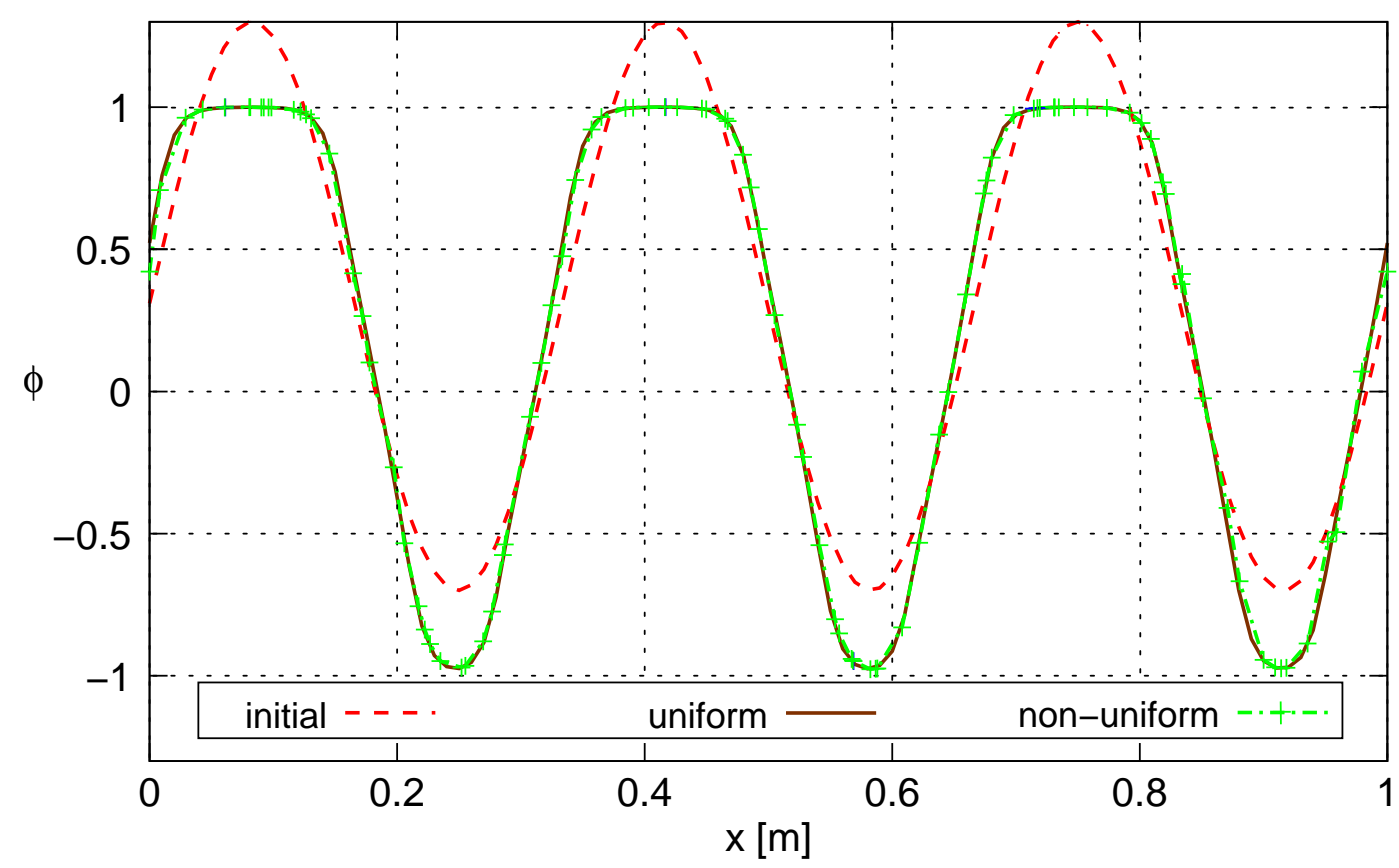

Figure 4: Travelling sinusoid solved by ADV+BRCP and initial condition, for uniform and non-uniform mesh spacings.

11 truecm The periodic advection problem is numerically solved with ADV+BRCP, considering a diffusive parameter $\kappa=2 h^{2}=2 \times 10^{-4} \mathrm{~m}$ combined with different penalty coefficient $M$ values. The results are plotted in Figure 5 together with the initial condition obtained from Equations (16) and (17) for a final time $t_{f}=2 \mathrm{~s}$, which corresponds to two laps around the periodic domain. The differences among penalizations $M=1, M=1000$ and $M=10000$ regarding the zeros position is that the lower penalty value gives the highest zeros delay, whereas $M=1000$ and $M=10000$ are closer to the position of the null LS function values $\phi=0$. By the other side, for $M=1000$ the bounds imposed to $\phi$, given by $\phi_{\text {ref }}=1$, are more respected than for $M=10000$, because a high $M$ value tends to get a curve attached to the advective solution $\phi^{0}$, which suffers a smoothing due to the proximity of peaks and the different sizes of the positive and negative regions of the LS function $\phi$. Considerations about the smoothing of the solution and the Values of $M$ between $M=1$ and $M=1000$ provide intermediate curves to those presented in Figure 5. The maximum zeros displacements $\Delta_{0}$ and maximum absolute variation of the length $\Delta l$ of LS positive or negative regions are summarized in Table 2, where the lowest variation in the length of a segment defined by a pair of zeros in the LS function, $\Delta l$, is registered for $M=1000$.

14 truecm

As a mesh convergence test, the same problem is solved with an element size of $h^{\prime}=h / 2=$ $0.005 \mathrm{~m}$ and time step $\Delta t=0.0025 \mathrm{~s}$ using ADV+BRCP with the same penalty coefficient values, $M=1, M=1000$ and $M=10000$, and renormalizing every $n_{\text {reno }}=20$ time steps. The diffusive parameter adopted is $\kappa^{\prime}=2\left(2 h^{\prime}\right)^{2}=2 \times 10^{-4} \mathrm{~m}$, i.e. the same numerical value than in the discretization with element length $h=0.01 \mathrm{~m}$, with the aim of reproducing the same transition width in both cases. After performing two laps around the periodic domain, the results show a similar behaviour as in Figure 5, though with lower zeros displacements with respect 


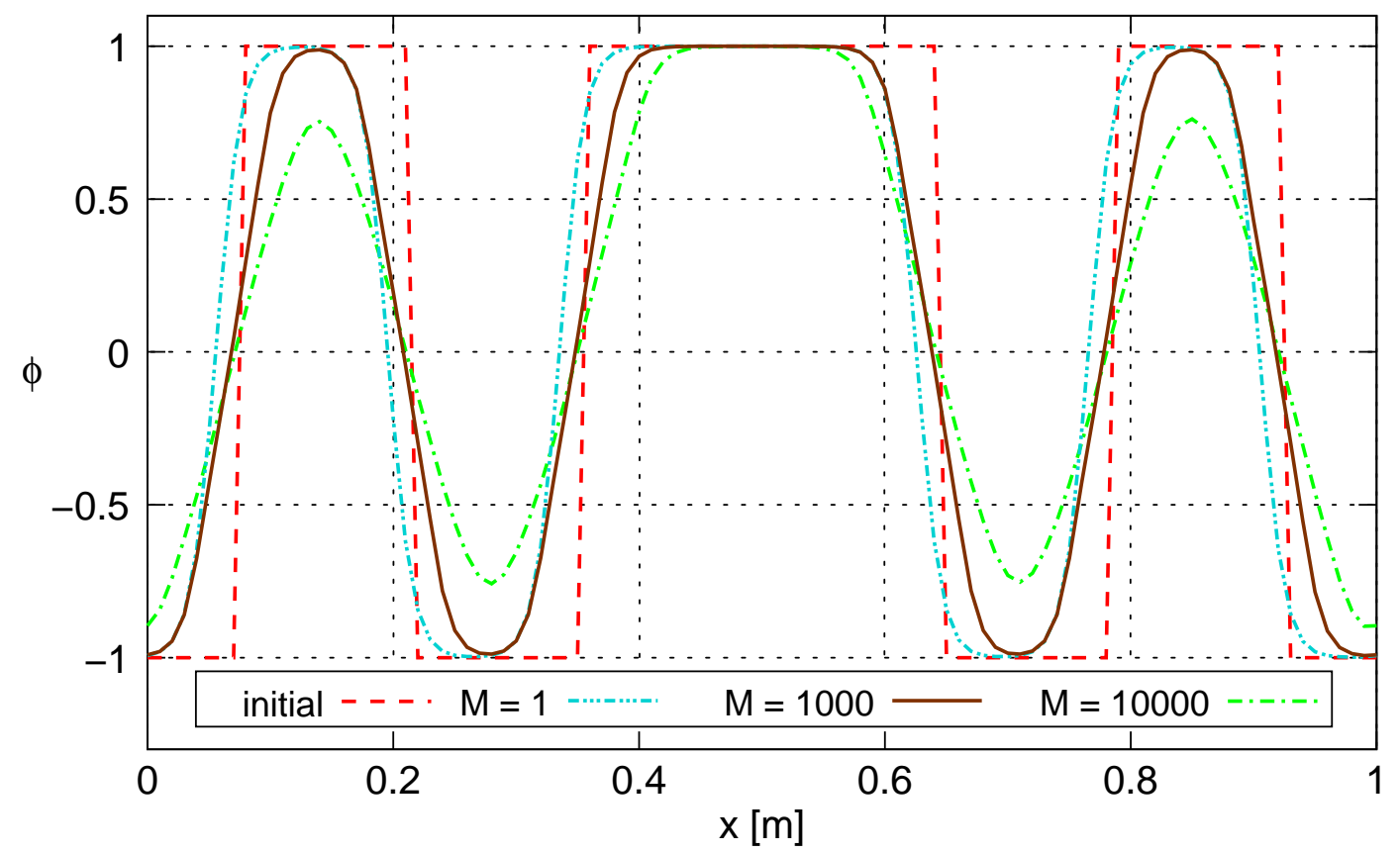

Figure 5: A normalized cardinal sine like function solved with ADV+BRCP, $h=0.01 \mathrm{~m}$, and different penalties $M$ after two laps.

\begin{tabular}{|l|c|c|c|c|} 
Element size & \multicolumn{2}{|c|}{$h=0.01 \mathrm{~m}$} & \multicolumn{2}{c|}{$h^{\prime}=0.005 \mathrm{~m}$} \\
Penalty & $\Delta_{0}[\mathrm{~m}]$ & $\Delta l[\mathrm{~m}]$ & $\Delta_{0}[\mathrm{~m}]$ & $\Delta l[\mathrm{~m}]$ \\
$M=1$ & $2.0 \times 10^{-2}$ & $8.5 \times 10^{-4}$ & $2.7 \times 10^{-3}$ & $3.0 \times 10^{-5}$ \\
$M=1000$ & $6.9 \times 10^{-3}$ & $1.7 \times 10^{-4}$ & $6.4 \times 10^{-3}$ & $9.2 \times 10^{-5}$ \\
$M=10000$ & $5.1 \times 10^{-3}$ & $1.8 \times 10^{-3}$ & $1.9 \times 10^{-3}$ & $1.2 \times 10^{-4}$
\end{tabular}

Table 2: Maximum zeros displacements $\Delta_{0}$ and variation of the distance between consecutive zeros $\Delta l$ for the cardinal sine like function after two laps solved with ADV+BRCP, and $h=0.01 \mathrm{~m}$.

to the theoretical solution and a better fit for the LS function proposed bounds, $\phi_{\text {ref }}$, and with a lower decrease of the peaks for a penalization of $M=10000$. Notice that the transition widths for $M=1$ and $M=1000$ values are the same as in the case with mesh size $h=0.01 \mathrm{~m}$ due to the use of the same value for $\kappa$ for both discretizations. The maximum zeros displacements $\Delta_{0}$ and maximum absolute variation of the length $\Delta l$ of LS positive or negative regions for a final time $t_{f}=2 \mathrm{~s}$ are included in Table 2, which are in all the cases lower than the corresponding ones determined with $h$, considering the $M$ values as reference and allowing the verification of the convergence according to the mesh size.

The imposition of initial conditions bounded to $\pm \phi_{\text {ref }}$ is recommended. These initial conditions could be obtained with the application of different functions over a field where $\phi=0$ is defined, such as $\tanh (\pi \phi)$, a distance function, or $\operatorname{sign}(\tilde{\phi})$. Nevertheless, as soon as the BRCP is performed, the $\phi$ field is smoothed, as seen in Figure 5. 


\subsection{Two-dimensional vortex example}

A typical test case for the advection-renormalization strategy consists of solving the deformation of a disc of radius $r=0.15 \mathrm{~m}$ with $\phi>1$ inside, centered in $\left(x_{c}, y_{c}\right)=(0.50,0.75) \mathrm{m}$ for a square domain with side length of $1 \mathrm{~m}$, i.e. $x$ and $y$-coordinates vary from 0 to $1 \mathrm{~m}$. This disc, represented exactly by the zero LS $\phi=0$, is submitted to a velocity field defined by a stream function

$$
\Xi=-\frac{1}{\pi} \sin ^{2}(\pi x) \sin ^{2}(\pi y)
$$

from which the velocity field over the domain is given by the following components,

$$
\begin{aligned}
& v_{x}=-\frac{\partial \Xi}{\partial y}=-\sin (2 \pi y) \sin ^{2}(\pi x) \\
& v_{y}=+\frac{\partial \Xi}{\partial x}=\sin (2 \pi x) \sin ^{2}(\pi y) .
\end{aligned}
$$

This example, or slightly different ones, is taken as reference for evaluating the performance of several interface-capturing methods such as LS Di Pietro et al. (2006); Enright et al. (2002); Gois et al. (2008); Olsson and Kreiss (2005), VOF Elias and Coutinho (2007) or hybrid ones Shin and Juric (2009). 12truecm

The selected variant is the application of the velocity field from Equations $(20,21)$ up to a final time $t_{\mathrm{f}}=5 \mathrm{~s}$. The results obtained by the FEM advection-renormalization strategy are compared to the position of some particles initially disposed over the contour of the circle and transported by the given velocity field. In this way, the comparison between the FEM resolution and the theoretical deformation allows the evaluation of the ability of the method for reproducing the disc deformation and the capabilities related to area preservation.

\section{4 truecm}

The results of this problem are obtained in the following ways:

A. advection (ADV) over a uniform tesselation of around 131000 linear triangular elements, with $N=256$ elements in each boundary side;

B. as in A, with SUPG stabilization.

C. $\mathrm{ADV}+\mathrm{BRCP}$, renormalizing every $n_{\text {reno }}=10$ time steps over the same discretization as A, with diffusivity $\kappa=2 h^{2}=3.06 \times 10^{-5} \mathrm{~m}^{2}$ and penalization $M=10000$;

D. ADV + BRCP for $N=512$ triangular elements by side, $n_{\text {reno }}=20$, with diffusivity $\kappa=2(2 h)^{2}=3.06 \times 10^{-5} \mathrm{~m}^{2}$ and penalization $M=10000$;

All the solutions for this case count on an initial field with linear variation from $\phi=-1$ to $\phi=1$, with an interface semi-width of $0.05 \mathrm{~m}$, i.e. around 13 times the element size for $h=1 / 256 \mathrm{~m}$. In all the cases, the Courant number is taken as $\mathrm{Co}=0.5$, which leads to a time step $\Delta t=1.95 \times 10^{-3} \mathrm{~s}$ in cases $\mathrm{A}, \mathrm{B}$ and $\mathrm{C}$, whereas $\Delta t=9.77 \times 10^{-4} \mathrm{~s}$ corresponds to case D. The time integration is made with an integration coefficient $\theta=0.5$ for the trapezoidal rule.

10 truecm The interface obtained through FEM, indicated with $\phi=0$, is represented in Figure 6 with continuous trace, whereas the reference particles are plot with crosses. This figure shows 

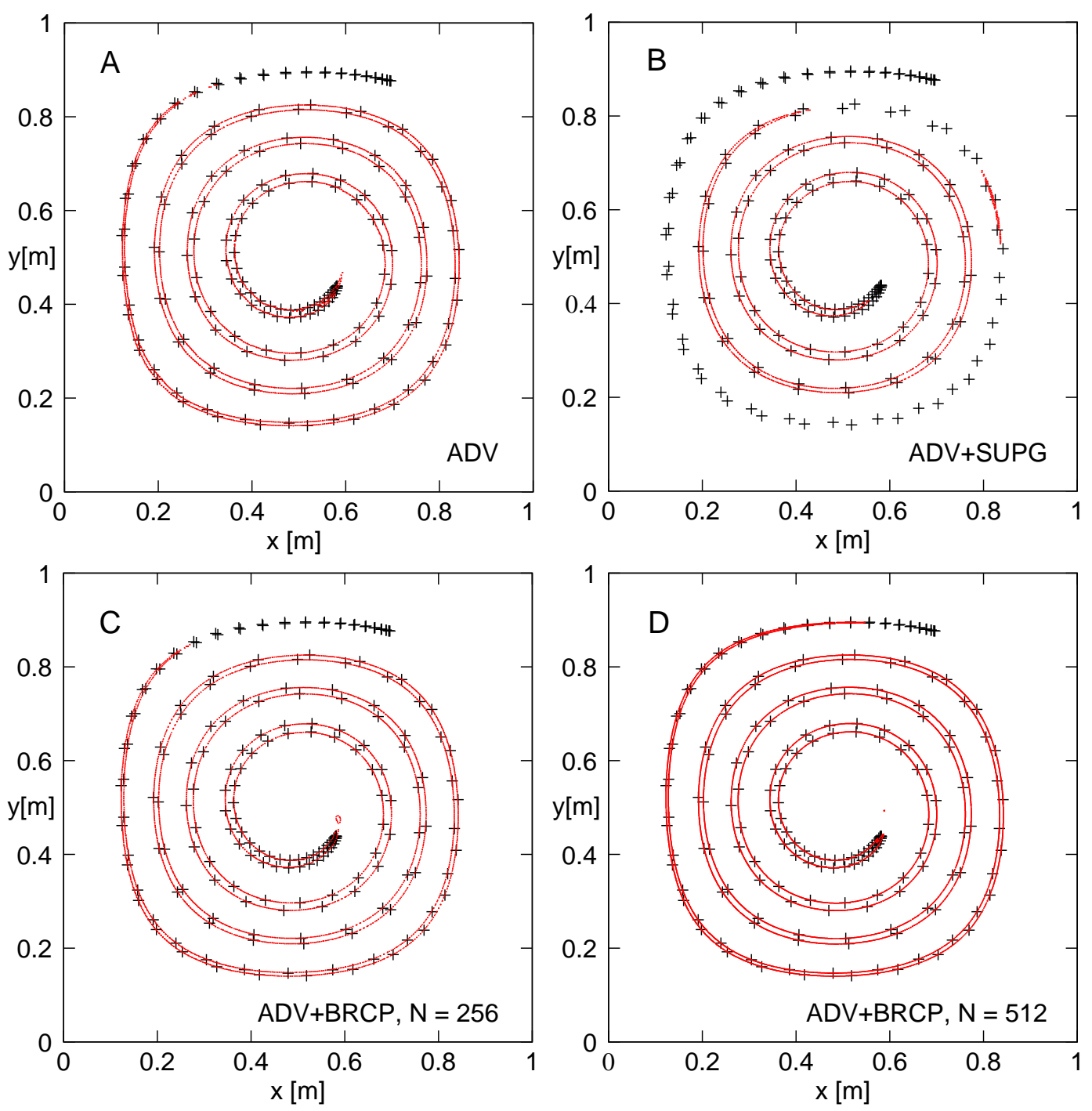

Exact $+\quad$ Numerical -

Figure 6: Curves of $\phi=0$ in $t_{\mathrm{f}}=5 \mathrm{~s}$ for the 2D vortex solved with ADV (A), ADV+SUPG (B), ADV+BRCP (C), and $\mathrm{ADV}+\mathrm{BRCP}$ in a refined domain (D).

little difference between the curves obtained in cases A and C, a higher performance in D, which is attributed to the refinement of the mesh, and an important loss of the positive LS function area, i.e. where $\phi>0$, in B.

The evolution of the positive LS function area is shown in Figure 7 for all the cases. In spite of the small differences between the results of cases $\mathrm{A}$ and $\mathrm{C}$ up to time $t=4 \mathrm{~s}$, the resolution in $\mathrm{C}$ shows less than $0.5 \%$ of area loss, the same order as in D, whereas in case $\mathrm{A}$ the positive area $(\phi>0)$ is diminished in 3\%, approximately. It is clear from Figure 6 that the area loss in $\mathrm{B}$ is much higher than those mentioned above, around $27 \%$.

As a reference, it should be mentioned that the results obtained by Olsson and Kreiss Olsson and Kreiss (2005) with a similar renormalization proposal showed higher mass loss and fragmentation of the $\phi>0$ area than case $\mathrm{C}$, for time $t=4 \mathrm{~s}$ and a $256^{2}$ elements mesh.

From another point of view, the analysis of the LS function profile along a section at $y=$ 


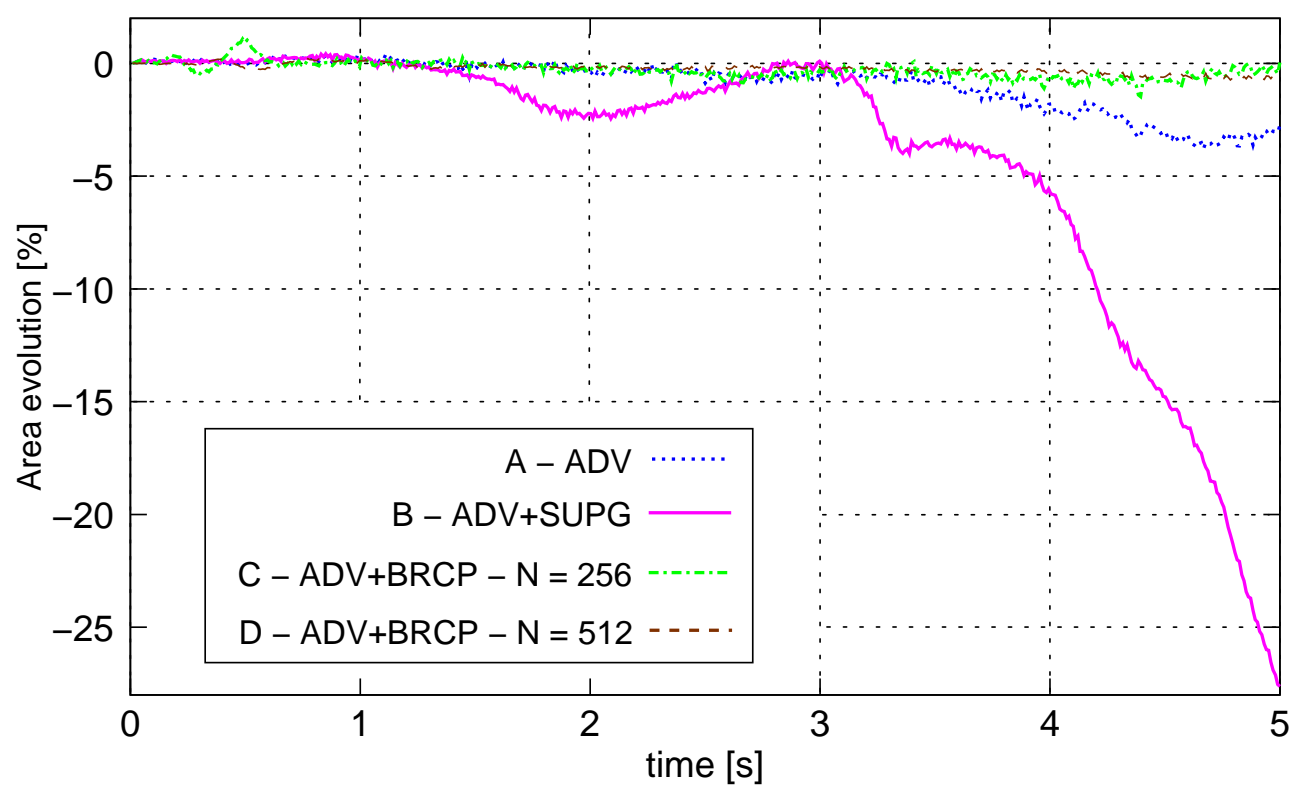

Figure 7: Area evolution of the region with positive LS function $(\phi>0)$ for the 2D vortex solved through ADV (A), ADV+SUPG (B) and ADV+BRCP for different mesh sizes (C,D).

$0.5 \mathrm{~m}$, represented in Figure 8, shows differences among the ADV solution in case A, with numerical instabilities, a smooth ADV+BRCP solution without numerical stabilization in the advection stage in $\mathrm{C}$, and a stabilized ADV solution in case $\mathrm{B}$, where the use of SUPG lead to the dissapearance of part of the area with $\phi>0$ due to the diffusion introduced by the SUPG method.

\subsection{Three-dimensional vortex example}

The three-dimensional extension of the two-dimensional vortex of Section 4.2 was proposed by LeVeque LeVeque (1996) and considered later by several authors such as Elias and Coutinho Elias and Coutinho (2007), Enright et al. Enright et al. (2002), Gois et al. Gois et al. (2008) and Shin and Juric Shin and Juric (2009), among others.

The example consists of the deformation of a sphere with radius $r_{e}=0.15 \mathrm{~m}$ defined by the LS corresponding to $\phi=0$, centered in coordinates $(0.35,0.35,0.35) \mathrm{m}$ of the unit cube, submitted to a velocity field given as follows,

$$
\left\{\begin{array}{llll}
v_{x}=2 \sin ^{2}(\pi x) & \sin (2 \pi y) & \sin (2 \pi z) & \cos \left(\frac{\pi t}{T_{e}}\right) \\
v_{y}=-\sin (2 \pi x) & \sin ^{2}(\pi y) & \sin (2 \pi z) & \cos \left(\frac{\pi t}{T_{e}}\right) \\
v_{z}=-\sin (2 \pi x) & \sin (2 \pi y) & \sin ^{2}(\pi z) & \cos \left(\frac{\pi t}{T_{e}}\right)
\end{array}\right.
$$

where the period is $T_{e}=3 \mathrm{~s}$. The proposed time evolution generates two contra-rotating vortices that produce a deformation of the region delimited by the interface $\phi=0$ between $t=0$ and $t=1.5 \mathrm{~s}$, resulting in a thin, squashed form. Then, the velocity field is inverted and the sphere should be recovered in $t=3 \mathrm{~s}$.

10 truecm

The FEM simulation is made over a $128^{3}$ hexahedral elements regular mesh, giving $h=$ $7.8 \times 10^{-3} \mathrm{~m}$ for the element size, with $n_{\text {reno }}=10$ time steps and a time step of $\Delta t=0.00195 \mathrm{~s}$. 


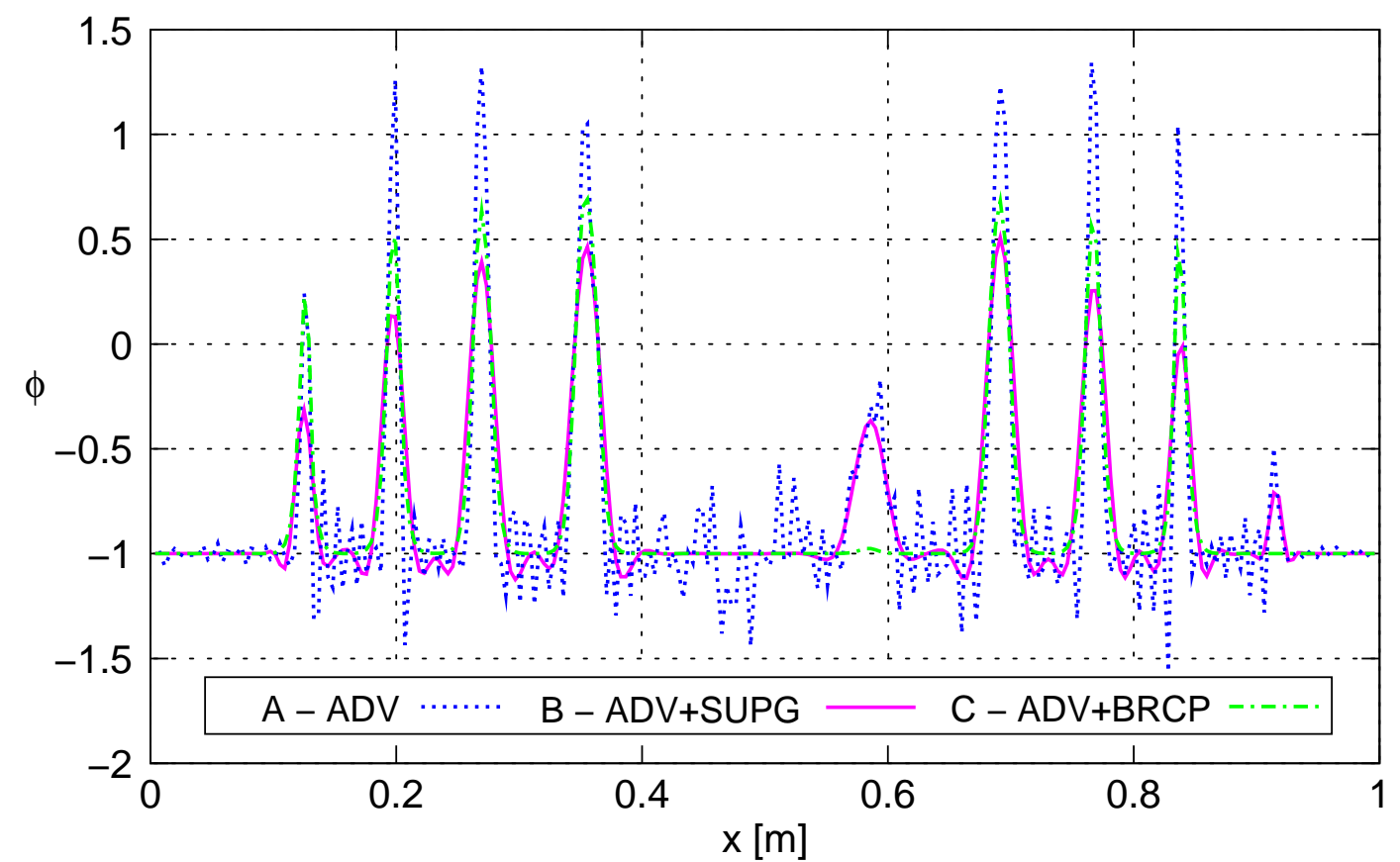

Figure 8: Section at $y=0.5 \mathrm{~m}$ for the $2 \mathrm{D}$ vortex problem in $t_{\mathrm{f}}=5 \mathrm{~s}$ for ADV (A), ADV+SUPG (B) and $\operatorname{ADV}+\mathrm{BRCP}(\mathrm{C})$.

For the BRCP instance, the diffusivity $\kappa=4 h^{2}=2.44 \times 10^{-4} \mathrm{~m}^{2}$ and the penalty coefficient $M=100000$ is adopted as a result of numerical experiments, from which the practical rule is proposing $M$ of $\mathcal{O}\left(10^{n_{d}+2}\right)$. The initial condition is proposed as a linear transition of $\phi$ across the interface.

Figure 9 shows the evolution of the interface $\phi=0$ in different time instants. Because of the periodic character of the velocity field, the shapes registered in $t=3 \mathrm{~s}$ and $t \approx 0$ should be equal, as well as $t=2.617 \mathrm{~s}$ and $t=0.390 \mathrm{~s}$, and the other corresponding pairs. A final volume loss of $5.4 \%$ is registered for the example solved under these conditions, and the evolution of this parameter is shown in Figure 10.

Similar spatial discretizations were employed for other authors for solving the 3D vortex. Those obtained by Enright et al. Enright et al. (2002) for a classical LS method with $100^{3}$ elements showed $80 \%$ of volume loss, while a particle LS for the same discretization of $100^{3}$ elements showed a $2.6 \%$ reduction of the volume. For VOF extensions, with a fully implicit parallel edge-based SUPG finite element formulation for the advection of the volume fraction, Elias and Coutinho Elias and Coutinho (2007) performed several tests with variants of the formulation over meshes with tetrahedral elements of typical size $h=7.5 \times 10^{-3}$ for a unit side cube. These last results presented a minimum volume variation of $-0.57 \%$ taking into account a mass conserving strategy, a maximum one of $-64 \%$ by adding a discontinuity capturing term to the resolution of the transport of the volume fraction, while combining both of the last improving strategies to the basic proposal, the volume variation is $+0.96 \%$.

10 truecm 

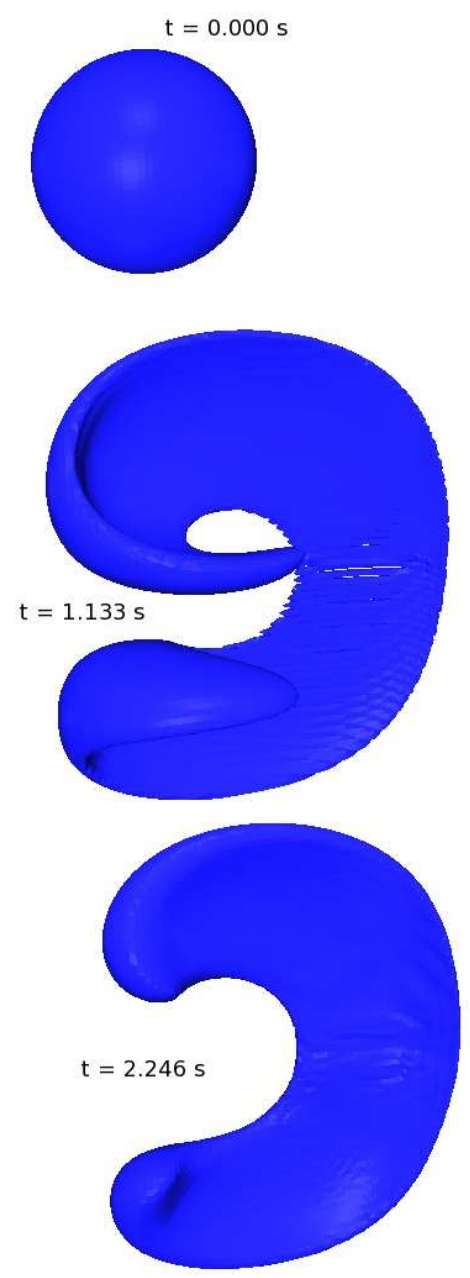

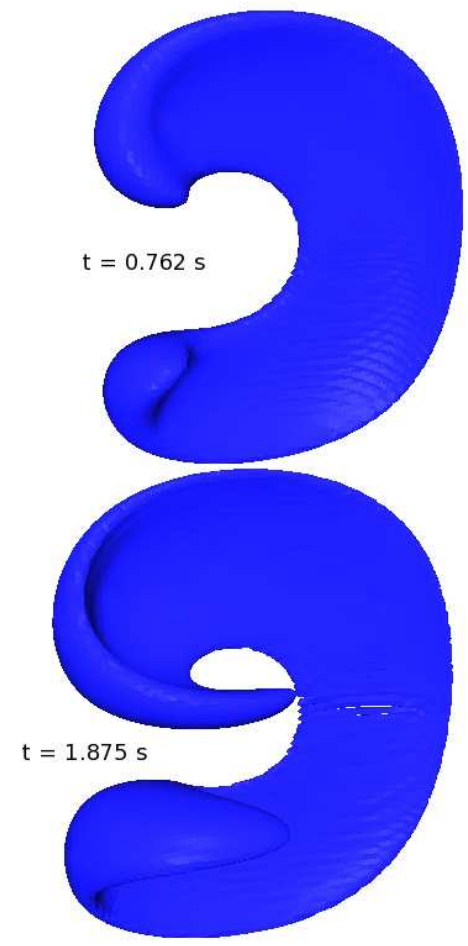

Figure 9: Surface defined by $\phi=0$ in different instants for the 3D vortex solved with ADV+BRCP.

\section{CONCLUSIONS}

A continuous operator, named BRCP, has been proposed for regularizing the LS function in one, two and three spatial dimensions by means of a finite element method. This operator avoids the degradation of some properties of the LS function, as the smoothness of the transition from one to another region, due to the advection of such field.

Two kinds of typical cases in one and two spatial dimensions have been solved by this procedure: advection and advection-renormalization problems, where the BRCP strategy is strongly tested in Section 4 and is compared in some cases to solutions obtained with advection and SUPG stabilized advection for the LS function field. These tests, together with the three dimensional example, allow the definition of ranges for the two parameters involved in the BRCP strategy, the diffusion $\kappa$ and the penalization $M$, related to the mesh size and the number of spatial dimensions involved, respectively. Advantages of the application of the renormalization procedure are shown especially in long time simulations, where the LS function profile is kept by BRCP, although this profile is not kept by either the advection or stabilized advection 


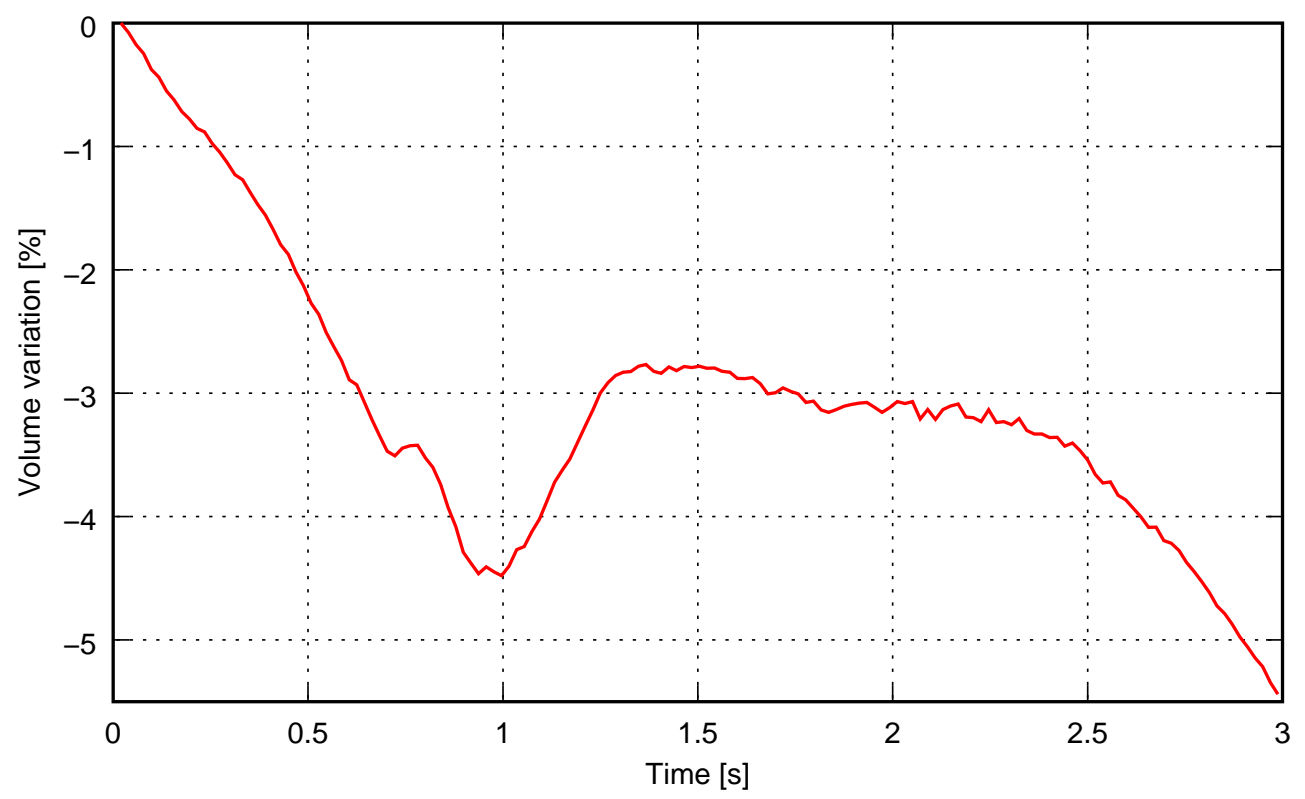

Figure 10: Shape volume evolution in time for the 3D vortex.

alternatives. Additionally, the renormalization procedure shows a numerical stabilizing effect over the results provided by the advection stage, avoiding the use of SUPG stabilization in that instance.

Further work would be focused on including a wider range of boundary conditions for the advection instance, and the coupling of this strategy with a fluid flow solver with the aim of modelling free surface and two-fluid flows cases.

This work has received financial support from Consejo Nacional de Investigaciones Científicas y Técnicas (CONICET, Argentina, grant PIP 5271/05), Universidad Nacional del Litoral (UNL, Argentina, grant CAI+D 2009-III-4-2), Agencia Nacional de Promoción Científica y Tecnológica (ANPCyT, Argentina, grants PICT 1506/06, PICT 1141/07) and was performed with the Free Software Foundation/ GNU-Project resources such as GNU/Linux OS, GNU/gcc and GNU/Octave, as well as other Open Source resources as Xfig, JabRef, ParaView and L $\mathrm{TT}_{\mathrm{E}} \mathrm{X}$.

\section{REFERENCES}

Balay S., Buschelman K., Eijkhout V., Gropp W.D., Kaushik D., Knepley M.G., McInnes L.C., Smith B.F., and Zhang H. PETSc users manual. Technical Report ANL-95/11 - Revision 3.0.0, Argonne National Laboratory, 2008.

Battaglia L. Stabilized Finite Elements for Free Surface Flows: Tracking and Capturing of Interface. Ph.D. thesis, Facultad de Ingeniería y Ciencias Hídricas, Universidad Nacional del Litoral, Santa Fe, Argentina, 2009. In Spanish, http://bibliotecavirtual.unl.edu.ar:8180/tesis/handle/1/115.

Battaglia L., D' Elía J., Storti M.A., and Nigro N.M. Numerical simulation of transient free surface flows using a moving mesh technique. ASME J. of Appl. Mech., 73(6):1017-1025, 2006. doi:10.1115/1.2198246.

Battaglia L., Storti M.A., and D' Elía J. An interface capturing finite element approach 
for free surface flows using unstructured grids. In Cardona, Storti, and Zuppa, editors, Mecánica Computacional, volume XXVII, pages 33-48. Asociación Argentina de Mecánica Computacional (AMCA), San Luis, Argentina, 10-13 November 2008. URL http://www.cimec.org.ar/ojs/index.php/mc/article/view/1396.

Battaglia L., Storti M.A., and D’Elía J. An interface capturing approach with bounded continuous renormalization for free surface flows. In García Bauza, Lotito, Parente, and Vénere, editors, Mecánica Computacional, volume XXVIII, pages 1423-1440. Asociación Argentina de Mecánica Computacional (AMCA), Tandil, Argentina, 3-6 November 2009. URL http://www.cimec.org.ar/ojs/index.php/mc/article/view/2809.

Brooks A. and Hughes T.J.R. Streamline upwind/Petrov-Galerkin formulations for convection dominated flows with particular emphasis on the incompressible Navier-Stokes equations. Comp. Meth. in Appl. Mech. Engr., 32(1-3):199-259, 1982. doi:10.1016/0045-7825(82) 90071-8.

Cirak F. and Radovitzky R. A Lagrangian-Eulerian shell-fluid coupling algorithm based on level sets. Computers \& Structures, 83(6-7):491-498, 2005. doi:10.1016/j.compstruc.2004. 03.085 .

Cruchaga M.A., Celentano D.J., and Tezduyar T.E. Collapse of a liquid column: numerical simulation and experimental validation. Comput. Mech., 39(4):453-476, 2007. doi:10.1007/ s00466-006-0043-z.

Di Pietro D.A., Lo Forte S., and Parolini N. Mass preserving finite element implementations of the level set method. Appl. Numerical Mathematics, 56(9):1179-1195, 2006. doi:10.1016/j. apnum.2006.03.003.

Donea J. and Huerta A. Finite Element Methods for Flow Problems. John Wiley \& Sons, 2003. doi:10.1002/0470013826.

Elias R. and Coutinho A.L.G.A. Stabilized edge-based finite element simulation of free-surface flows. Int. J. for Num. Meth. in Fluids, 54(6-8):965-993, 2007. doi:10.1002/fld.1475.

Elias R., Martins M.A.D., and Coutinho A.L.G.A. Simple finite element-based computation of distance functions in unstructured grids. Int. J. for Num. Meth. in Engr., 72(9):1095-1110, 2007. doi:10.1002/nme.2079.

Enright D., Fedkiw R., Ferziger J., and Mitchell I. A hybrid particle level set method for improved interface capturing. J. of Comput. Physics, 183(1):83-116, 2002. doi:10.1006/ jcph.2002.7166.

Gloger O., Ehrhardt M., Dietrich T., Hellwich O., Graf K., and Nagel E. A threestepped coordinated level set segmentation method for identifying atherosclerotic plaques on MR-images. Comm. in Num. Meth. in Engineering, 25(6):615-638, 2009. doi:10.1002/cnm.1213.

Gois J.P., Nakano A., Nonato L.G., and Buscaglia G.C. Front tracking with moving-leastsquares surfaces. J. of Comput. Physics, 227(22):9643-9669, 2008. doi:10.1016/j.jcp.2008. 07.013.

Hettich T. and Ramm E. Interface material failure modeled by the extended finite-element method and level sets. Computer Methods in Applied Mechanics and Engineering, 195(3740):4753-4767, 2006. ISSN 0045-7825. doi:10.1016/j.cma.2005.09.022.

Hirt C.W. and Nichols B.D. Volume of fluid (VOF) method for the dynamics of free boundaries. J. of Comput. Physics, 39(1):201-225, 1981. doi:10.1016/0021-9991(81)90145-5. 
Huerta A. and Liu W.K. Viscous flow with large free surface motion. Comp. Meth. in Appl. Mech. and Engr., 69(3):277-324, 1988. doi:10.1016/0045-7825(88)90044-8.

Hughes T.J.R., Liu W.K., and Zimmermann T.K. Lagrangian-Eulerian finite element formulation for incompressible viscous flows. Comp. Meth. in Appl. Mech. and Engr., 29(3):329-349, 1981. doi:10.1016/0045-7825(81)90049-9.

Idelsohn S.R., Oñate E., and Del Pin F. The Particle Finite Element Method: a powerful tool to solve incompressible flows with free-surfaces and breaking waves. Int. J. for Num. Meth. in Engr., 61(7):964-984, 2004. doi:10.1002/nme.1096.

LeVeque R.J. High-resolution conservative algorithms for advection in incompressible flow. SIAM J. on Numerical Analysis, 33(2):627-665, 1996. doi:10.1137/0733033.

Logan J.D. An introduction to nonlinear partial differential equations. Pure and Applied Mathematics. Wiley-Interscience, 1994. doi:10.1002/9780470287095.

Maitre E., Milcent T., Cottet G.H., Raoult A., and Usson Y. Applications of level set methods in computational biophysics. Mathematical and Computer Modelling, 49(11-12):2161-2169, 2009. doi:10.1016/j.mcm.2008.07.026.

Marchandise E. and Remacle J.F. A stabilized finite element method using a discontinuous level set approach for solving two phase incompressible flows. J. of Comput. Physics, 219(2):780800, 2006. doi:10.1016/j.jcp.2006.04.015.

MPI. Message Passing Interface. http://www.mpi-forum.org, 2009.

Mut F., Buscaglia G.C., and Dari E.A. New mass-conserving algorithm for level set redistancing on unstructured meshes. J. of Appl. Mech., 73(6):1011-1016, 2006. doi:10.1115/1.2198244.

Olsson E. and Kreiss G. A conservative level set method for two phase flow. J. of Comput. Physics, 210(1):225-246, 2005. doi:10.1016/j.jcp.2005.04.007.

Olsson E., Kreiss G., and Zahedi S. A conservative level set method for two phase flow II. J. of Comput. Physics, 225(1):785-807, 2007. doi:10.1016/j.jcp.2006.12.027.

Osher S. and Fedkiw R.P. Level set methods: An overview and some recent results. J. of Comput. Physics, 169(2):463-502, 2001. doi:10.1006/jcph.2000.6636.

Osher S. and Sethian J.A. Fronts propagating with curvature-dependent speed: Algorithms based on Hamilton-Jacobi formulations. J. of Comput. Physics, 79(1):12-49, 1988. doi: 10.1016/0021-9991(88)90002-2.

PETSc-FEM. A general purpose, parallel, multi-physics FEM program. 2009. GNU General Public License (GPL), http://www.cimec.org.ar/petscfem.

Scardovelli R. and Zaleski S. Direct numerical simulation of free-surface and interfacial flow. Annual Reviews of Fluid Mechanics, 31:567-603, 1999. doi:10.1146/annurev.fluid.31.1.567.

Sethian J.A. A fast marching level set method for monotonically advancing fronts. National Academy of Sciences, 1995. doi:10.1073/pnas.93.4.1591.

Shin S. and Juric D. A hybrid interface method for three-dimensional multiphase flows based on front tracking and level set techniques. Int. J. for Num. Meth. in Fluids, 60(7):753-778, 2009. doi:10.1002/fld.1912.

Shyy W., Udaykumar H.S., Rao M.M., and Smith R.W. Computational Fluid Dynamics with Moving Boundaries. Taylor and Francis, 1996. doi:10.2514/2.7524.

Sussman M. and Puckett E.G. A coupled level set and volume-of-fluid method for computing 3D and axisymmetric incompressible two-phase flows. J. of Comput. Physics, 162(2):301- 
337, 2000. doi:10.1006/jcph.2000.6537.

Sussman M. and Smereka P. Axisymmetric free boundary problems. J. of Fluid Mechanics, 341:269-294, 1997. doi:10.1017/S0022112097005570.

Tezduyar T.E. Interface-tracking and interface-capturing techniques for finite element computation of moving boundaries and interfaces. Comp. Meth. in Appl. Mech. and Engr. 195(2324):2983-3000, 2006. doi:10.1016/j.cma.2004.09.018.

Tezduyar T.E. and Osawa Y. Finite element stabilization parameters computed from element matrices and vectors. Comp. Meth. in Appl. Mech. and Engr., 190(3-4):411-430, 2000. doi: 10.1016/S0045-7825(00)00211-5. 